\title{
INFLUÊNCIA DO ESPAÇAMENTO ENTRE ASPERSORES NA UNIFORMIDADE DE DISTRIBUIÇÃO DE ÁGUA ACIMA E ABAIXO DA SUPERFÍCIE DO SOLO
}

\author{
Roberto Rezende ${ }^{1}$, José Antonio Frizzone ${ }^{2}$, Antônio Carlos Andrade Gonçalves ${ }^{3}$ \\ e Paulo Sérgio Lourenço de Freitas ${ }^{4}$
}

\begin{abstract}
RESUMO
A uniformidade de aplicação de água por um sistema de irrigação por aspersão convencional, foi estudada acima e abaixo da superfície do solo. Foram adotados dois espaçamentos entre aspersores e coeficientes estatísticos de uniformidade empregados para a realização de comparações quantitativas. Os resultados obtidos mostram que a redistribuição de água no solo promove uniformidade da umidade do solo maior que a identificada para a lâmina aplicada. $\mathrm{O}$ maior espaçamento entre aspersores promoveu menor uniformidade, acima e abaixo da superfície; no entanto, a uniformidade da umidade do solo foi alta o bastante para atender aos critérios usualmente adotados na irrigação por aspersão, ao contrário do identificado acima da superfície.
\end{abstract}

Palavras-chave: irrigação, aspersão, uniformidade de distribuição

\section{THE INFLUENCE OF SPRINKLER SPACING ON THE DISTRIBUTION UNIFORMITY OF WATER ABOVE AND BELOW THE SOIL SURFACE}

\begin{abstract}
The application uniformity of water, below and above the soil surface, by a conventional sprinkler irrigation system was studied. Two sprinkler spacings were tested and statistical uniformity coefficients were used to make quantitative comparisons. The results obtained indicated that the redistribution of soil water promotes soil moisture uniformity greater than that identified for the depth of water applied. The larger sprinkler spacing resulted in lower uniformity below and above the soil surface, but soil moisture uniformity remained high enough to meet the usual criterias adopted in sprinkler irrigation, which was not the case above surface.
\end{abstract}

Key words: Irrigation, sprinkling, distribution uniformity

\section{INTRODUÇÃO}

A irrigação é uma operação agrícola que tem como finalidade básica colocar água à disposição da planta mantendo o solo com umidade adequada utilizando, para isto, diferentes métodos de irrigação.
Os diversos sistemas de irrigação têm sido avaliados pela uniformidade da água aplicada na superfície do solo, porém a planta retira água do volume de solo no qual o seu sistema radicular se encontra; portanto, pode-se supor que a uniformidade de distribuição da água no perfil do solo é mais importante que na sua superfície.

\footnotetext{
${ }^{1}$ Aluno de Doutorado no CPGID - ESALQ/USP. Prof. Assistente IV - UEM, Maringá, PR. Av. Pádua Dias 11, Departamento de Engenhari a Rural, Piracicaba, SP. CEP: 13.418-900

2 Prof. Associado - DER- ESALQ/USP

${ }^{3}$ Prof. Adjunto - Universidade Estadual de Maringá - UEM, Maringá, PR. E-mail: acagonca@ wnet.com.br

${ }^{4}$ Prof. Assistente IV - UEM, Maringá, PR. Aluno de Doutorado em Eng. Agrícola, DEA - UFV, Viçosa, MG
} 
Uma vez que a água tende a se movimentar das regiões de maior para as de menor umidade, a sua redistribuição no perfil do solo deve contribuir para aumentar a uniformidade, em relação à uniformidade medida na superfície.

Para se projetar sistemas de irrigação são utilizados dados de uniformidade de distribuição medidos na superfície, os quais subestimam a uniformidade da umidade no perfil do solo. Este procedimento não é o mais adequado quando o objetivo é o dimensionamento do sistema mais econômico.

Hart \& Reynolds (1965) usando um modelo matemático, estudaram a influência de diversas variáveis de irrigação na redistribuição de água dentro do solo e concluíram que a avaliação de sistemas de aspersão, pelas medidas de superfície, subestima a sua uniformidade, o que a torna inadequada se o objetivo do projeto é resultar no sistema mais econômico.

De acordo com Lopez (1975) o elemento climático mais importante na aspersão é o vento. Deve-se ter informações precisas de sua velocidade, que exerce papel significativo na eficiência de aplicação, e da sua direção, que deve ser levada em conta na disposição das tubulações. $\mathrm{O}$ autor esclarece que alta temperatura e baixa umidade relativa do ar diminuem a eficiência do sistema, aumentando as perdas por evaporação.

Coletando água em pluviômetros, Christiansen (1942) estimou as perdas por evaporação na irrigação por aspersão e concluiu que elas estiveram entre 10 e $42 \%$ para os testes realizados à tarde e próximo de $4 \%$ para aqueles conduzidos pela manhã. Mesmo não tendo realizado estudos para diferentes condições de clima, concluiu que as perdas são mais elevadas em dias com ventos fortes, quentes e secos. Keller (1984) cita que em condições de baixa demanda evaporativa as perdas por evaporação e arrastamento estão entre 5 e $10 \%$, chegando a valores entre 30 e $40 \%$, sob condições de alta demanda.

Nenhum sistema de irrigação é capaz de aplicar água com perfeita uniformidade. Em geral, o aumento da uniformidade de distribuição da água requer investimentos na melhoria do sistema, em manutenção e em mão-de-obra, para o manejo racional da irrigação (Heermann et al., 1992).

Conforme relatam alguns pesquisadores (Peri et al.,1979; Walker, 1979) a aplicação de água por qualquer sistema de irrigação é naturalmente desuniforme. Em geral, para um método particular de irrigação, a alta uniformidade de aplicação somente pode ser alcançada com aumento nos gastos em capital ou nos custos de operação. Esses autores afirmam que, sob o aspecto econômico, alguns níveis particulares de desuniformidade podem ser aceitáveis para cada tipo de irrigação. Ao irrigante, faculta-se escolher entre aplicar água suficiente para que a lâmina mínima seja igual à lâmina real necessária (aplicando excesso de água em parte da área) ou aplicar uma lâmina mínima menor, permitindo que parte da área seja deficientemente irrigada (provocando redução na produção).

Tarjuelo et al. (1996) salientam que os benefícios econômicos da irrigação aumentam em função do aumento da uniformidade de distribuição, independentemente do custo da água.

Mantovani et al. (1995) mostraram que a relação ótima entre lâmina de irrigação e lâmina de água necessária à cultura do milho depende da uniformidade de distribuição de água e da relação entre o preço do produto e o custo da água.

Vários coeficientes de uniformidade têm caracterizado a distribuição da água em sistemas de irrigação por aspersão. Christiansen (1942) foi o primeiro pesquisador a estudar a uniformidade de distribuição em aspersão, quantificando-a através de um índice estatístico; denominado coeficiente de uniformidade de Christiansen (CUC) este índice faz uso do desvio médio absoluto, para expressar a dispersão das lâminas e pode ser obtido pela equação:

$$
\mathrm{CUC}=100\left(1-\frac{\sum_{\mathrm{i}=1}^{\mathrm{n}}\left|\mathrm{X}_{\mathrm{i}}-\overline{\mathrm{X}}\right|}{\mathrm{n} \overline{\mathrm{X}}}\right)
$$

em que :

CUC - coeficiente de uniformidade de Christiansen, em \%

$\mathrm{X}_{\mathrm{i}}$ - lâmina coletada no pluviômetro i, mm

$\overline{\mathrm{X}} \quad$ - lâmina média coletada, $\mathrm{mm}$

$\mathrm{n}$ - número de coletores, adimensional

A ABNT (1985) recomenda, no seu projeto de norma 12:02.08-005, a análise da distribuição de água por meio da uniformidade na porção da área irrigada, que recebe menos água. O coeficiente de uniformidade de distribuição (CUD) é definido como a medida da distribuição da água que relaciona a quarta parte da área irrigada total, que recebe menos água, com a lâmina média aplicada. Caso toda a área receba no mínimo a lâmina real necessária, um baixo valor de CUD indica excessiva perda por percolação. Esse coeficiente pode ser obtido por meio da equação:

$$
\mathrm{CUD}=\frac{\mathrm{X}_{25}}{\overline{\mathrm{X}}} 100
$$

em que :

CUD - coeficiente de uniformidade de distribuição, em \%

$\mathrm{X}_{25}$ - média de $25 \%$ do total de pluviômetros, com as menores lâminas, em mm.

De acordo com Scaloppi (1985) a quantidade total de água a ser aplicada por meio de um sistema de irrigação por aspersão depende da quantidade real de água requerida e da eficiência de aplicação. A quantidade de água requerida, geralmente expressa como lâmina de irrigação, é determinada em função do déficit de água disponível no solo, até a profundidade definida pelo sistema radicular da cultura; eventuais exigências de lixiviação para remoção do excesso de sais solúveis da zona radicular também devem ser consideradas.

Jensen et al. (1967) afirmam que, conhecendo-se o perfil de distribuição dos aspersores, pode-se avaliar a adequação de uma irrigação, ou mesmo ajustar a sua duração, obtendo-se a lâmina desejada. Por razões econômicas, parte da área pode ser subirrigada, enquanto o restante da área recebe irrigação adequada. A porcentagem da área sub-irrigada depende do valor econômico e da sensibilidade das plantas à deficiência de água ou excesso.

Rezende et al. (1992) analisaram uniformidade de distribuição acima e abaixo da superfície do solo irrigado por pivô-central, utilizando os coeficientes CUC e CUD e concluíram que, em todas as profundidades, os coeficientes de uniformidade foram superiores aos obtidos acima da superfície do solo. Os valores dos coeficientes de uniformidade aumentaram com o tempo em todas as profundidades. Os altos valores alcançados pelos coeficientes de uniformidade abaixo da superfície do solo mostraram que a uniformidade abaixo da superfície é sempre maior que a uniformidade acima.

Paiva (1980) trabalhou com um sistema de irrigação por aspersão convencional, para analisar o efeito da uniformidade de aplicação sobre a uniformidade da umidade no perfil do 
solo, após a redistribuição. Após o fim da irrigação, os coeficientes de uniformidade aumentaram ao longo do tempo, em todas as profundidades estudadas; nas maiores profundidades, a distribuição foi mais uniforme, mesmo nos ensaios onde a uniformidade de água aplicada na superfície foi muito baixa. Em curtos intervalos de tempo, os coeficientes de uniformidade de umidade alcançaram altos valores.

Perrens (1984) relata que a intensidade de redistribuição da água no solo está inteiramente relacionada à textura. Avaliandose a uniformidade de umidade a $0,50 \mathrm{~m}$ de profundidade, durante o processo de redistribuição da água no solo, para um período de dois dias, a uniformidade aumentou de $60 \%$ para $67 \%$ em um solo de textura arenosa e de $60 \%$ para $73 \%$ em um solo tipo areno-siltoso.

O presente trabalho teve como objetivo avaliar, acima e abaixo da superfície do solo, a uniformidade de distribuição de água aplicada por um sistema de irrigação por aspersão convencional, segundo dois espaçamentos entre aspersores. Coeficientes estatísticos foram usados para quantificar a uniformidade de aplicação de água na superfície e a uniformidade de distribuição de água no perfil do solo.

\section{MATERIAL E MÉTODOS}

$\mathrm{Na}$ área experimental de irrigação do Departamento de Agronomia da Universidade Estadual de Maringá, Centro Técnico de Irrigação, foi conduzido o presente trabalho, no segundo semestre de 1995.

Em um período anterior aos ensaios, foi implantada a cultura do feijão, variedade IAPAR-14, na área selecionada para a condução do experimento. Esta cultura foi conduzida de maneira tradicional, no que diz respeito ao preparo do solo, semeadura, adubação e tratos fitossanitários.

As irrigações foram realizadas tendo-se como base as leituras de tensiômetros instalados na parcela experimental, nas profundidades de 0,20 e $0,40 \mathrm{~m}$, utilizando-se como limite o potencial mátrico de $-0,05 \mathrm{MPa}$.

Para a realização do experimento foram coletadas amostras de solo após seis irrigações efetuadas na cultura do feijoeiro.

\section{Sistema de irrigação}

O sistema de irrigação por aspersão utilizado foi composto de quatro linhas laterais adjacentes, com quatro aspersores por linha, funcionando simultaneamente à mesma pressão, ajustada através de válvulas de gaveta dispostas nos tubos de elevação dos aspersores.

As linhas laterais foram compostas com tubos de PVC de $50 \mathrm{~mm}$ de diâmetro, com comprimento de $36 \mathrm{~m}$ para um ensaio (espaçamento de $12 \mathrm{~m}$ ) e de $54 \mathrm{~m}$ (espaçamento de $18 \mathrm{~m}$ ) para o outro. As ligações entre os tubos e os aspersores (tubo de elevação) foram feitas com tubos de PVC de $25 \mathrm{~mm}$ de diâmetro e de $1,0 \mathrm{~m}$ de comprimento; nesses tubos foram instalados manômetros de "Bourdon", para monitorar a pressão em cada aspersor. Os aspersores utilizados foram da marca Fabrimar, modelo A232, com diâmetro de bocais de 5,6 x 3,2mm, operando a pressão de serviço de $350 \mathrm{kPa}$.

Trabalhou-se com dois espaçamentos entre aspersores, com a finalidade de se obter altos e baixos coeficientes de uniformidade de distribuição na superfície do solo, possibilitando a comparação com coeficientes abaixo da superfície.

\section{Condições ambientais}

Para se avaliar as possíveis influências do ambiente, foram caracterizadas as condições climáticas em função da velocidade do vento, umidade relativa do ar e temperatura, obtidos próximo da área dos ensaios, no posto meteorológico do Centro Técnico de Irrigação. A umidade relativa do ar foi obtida por meio de um termohigrógrafo e a velocidade do vento foi medida por meio de um anemômetro de conchas totalizador, instalado a $2 \mathrm{~m}$ de altura em relação à superfície do solo; a leitura desse instrumento foi feita a cada 15 minutos, durante a realização dos ensaios obtendo-se, assim, a velocidade média do vento para cada ensaio. Foi utilizado um par de termômetros graduados a cada $0,2^{\circ} \mathrm{C}$, com a finalidade de se caracterizar a variação da temperatura.

\section{Distribuição superficial da água aplicada}

A área entre aspersores foi subdividida em quadrículas de três metros de lado; no centro de cada quadrícula foi colocado um coletor, com o propósito de receber parte da água aplicada, dado este necessário aos cálculos dos parâmetros estatísticos que caracterizam a distribuição de água do sistema de irrigação. Foram usados recipientes com $0,10 \mathrm{~m}$ de diâmetro, fixados em hastes metálicas, de forma a ficarem a uma altura de $0,40 \mathrm{~m}$ da superfície do solo.

\section{Distribuição de umidade abaixo da superfície do solo}

A determinação da uniformidade de distribuição de água abaixo da superfície do solo foi realizada a partir de medidas de umidade realizadas na camada de 0,15 a $0,20 \mathrm{~m}$, em dois pontos situados a $0,30 \mathrm{~m}$ de cada coletor. Amostras de solo foram coletadas ao lado de cada coletor, antes de se realizar cada uma das irrigações; doze horas após o final da irrigação, nova amostragem foi realizada e a umidade foi determinada pelo método gravimétrico.

\section{Coeficientes de uniformidade}

As medidas de uniformidade de distribuição expressam a variabilidade da lâmina de irrigação aplicada nas áreas adjacentes aos aspersores, isto é, mostram a dispersão dos valores obtidos em relação à lâmina média. Neste trabalho, os coeficientes de uniformidade foram calculados para as distribuições superficial e subsuperficial, na profundidade e no tempo já mencionados. Antes e após cada irrigação, foram calculados os coeficientes de uniformidade de Christiansen e de uniformidade de distribuição.

\section{RESULTADOS E DISCUSSÃO}

As condições climáticas reinantes durante os ensaios realizados foram relativamente estáveis, não sendo possível detectar seus efeitos sobre os coeficientes de uniformidade, tendo-se como média de velocidade do vento $0,70 \mathrm{~m} / \mathrm{s}$, média de umidade relativa $63 \%$ e temperatura média $24,5^{\circ} \mathrm{C}$.

$\mathrm{Na}$ Tabela 1 são apresentados os valores dos coeficientes de uniformidade determinados acima e abaixo da superfície do solo, para espaçamento entre aspersores de $12 \times 12 \mathrm{~m}$; nesta tabela, pode-se observar que a variação dos coeficientes de uniformidade entre as irrigações é pequena, em todas as condições estudadas, o que é expresso por valores de coeficientes de variação $(\mathrm{CV})$ inferiores a $1 \%$ mostrando que o padrão de 
aplicação de água pouco se alterou com o passar do tempo, induzindo a comportamento semelhante da umidade do solo. Os altos valores dos coeficientes mostram que foram uniformes as distribuições de água nas irrigações realizadas.

Todos os valores médios dos coeficientes de uniformidade de Christiansen são superiores ao valor mínimo aceitável preconizado na literatura $(80 \%)$ para o desempenho normal do sistema de aspersão (Olitta,1981).

De acordo com a Tabela 1, todos os valores dos coeficientes de uniformidade determinados abaixo da superfície foram superiores aos determinados acima da superfície do solo. Tal constatação está relacionada com a redistribuição de água no solo. A tendência da água se movimentar de regiões mais úmidas para aquelas de menor umidade promove, após certo tempo, maior uniformidade da umidade.

Abaixo da superfície o CUC pouco se alterou, antes e após as irrigações, mostrando a pouca sensibilidade deste coeficiente a valores extremos. Os valores médios não diferem estatisticamente, pelo teste " $t$ ", a nível de 5\% de significância. As demais médias, apresentadas na Tabela 1, diferem a nível de 5\%. O CUD, sendo calculado com base nos menores valores, é mais sensível a valores extremos e registra tendência de redução de valores entre as duas condições, mostrando maior irregularidade da umidade do solo após cada irrigação.

Tabela 1. Valores dos coeficientes de uniformidade* determinados acima e abaixo da superfície do solo para espaçamentos entre aspersores de $12 \times 12 \mathrm{~m}$

\begin{tabular}{|c|c|c|c|c|c|c|}
\hline \multirow[t]{6}{*}{ Irrigação } & \multicolumn{6}{|c|}{ Coeficientes de uniformidade (\%) } \\
\hline & \multirow{2}{*}{\multicolumn{2}{|c|}{$\begin{array}{l}\text { Antes da } \\
\text { Irrigação }\end{array}$}} & \multirow{2}{*}{\multicolumn{4}{|c|}{$\begin{array}{l}\text { Depois da } \\
\text { Irrigação }\end{array}$}} \\
\hline & & & & & & \\
\hline & \multirow{2}{*}{\multicolumn{2}{|c|}{$\begin{array}{l}\text { Abaixo da } \\
\text { Superfície }\end{array}$}} & \multirow{2}{*}{\multicolumn{2}{|c|}{$\begin{array}{l}\text { Acima da } \\
\text { Superfície }\end{array}$}} & \multirow{2}{*}{\multicolumn{2}{|c|}{$\begin{array}{l}\text { Abaixo da } \\
\text { Superfície }\end{array}$}} \\
\hline & & & & & & \\
\hline & CUC & CUD & CUC & CUD & CUC & CUD \\
\hline 1 & 95,43 & 93,83 & 89,06 & 81,90 & 96,16 & 90,90 \\
\hline 2 & 96,10 & 94,40 & 87,78 & 82,73 & 95,43 & 92,94 \\
\hline 3 & 96,52 & 94,53 & 88,30 & 83,07 & 95,62 & 91,58 \\
\hline 4 & 96,78 & 94,18 & 88,00 & 82,32 & 94,98 & 90,96 \\
\hline 5 & 95,93 & 93,96 & 88,23 & 81,62 & 96,09 & 92,47 \\
\hline 6 & 95,96 & 94,78 & 88,01 & $8 \mathrm{I}, 20$ & 94,36 & 92,63 \\
\hline$\overline{\mathrm{X}}$ & $96,12 \mathrm{a}$ & $94,28 b$ & $88,23 \mathrm{c}$ & $82,14 d$ & $95,44 \mathrm{a}$ & $91,83 \mathrm{e}$ \\
\hline $\mathrm{CV}$ & 0,50 & 0,38 & 0,51 & 0,72 & 0,72 & 0,96 \\
\hline
\end{tabular}

Na Tabela 2 são apresentados os valores dos coeficientes de uniformidade determinados acima e abaixo da superfície do solo, para espaçamento entre aspersores de $18 \times 18 \mathrm{~m}$. Observa-se que os coeficientes de uniformidade de distribuição variaram pouco entre irrigações; no entanto, esta variação foi maior quando comparada com os dados da Tabela 1, o que demonstra que a irrigação feita com uma superposição menor sofreu mais a ação de fatores não controlados, como a velocidade do vento, promovendo maiores diferenças entre irrigações. As irregularidades na aplicação de água, embora suavizadas no perfil do solo, promovem irregularidades na umidade abaixo da superfície. A comparação entre os coeficientes de variação abaixo da superfície, entre os espaçamentos de $12 \mathrm{~m}$ e de $18 \mathrm{~m}$, demostra este fato.

Os baixos valores dos coeficientes acima da superfície, depois das irrigações, mostram que estas foram realizadas com baixa uniformidade. A Tabela 2 permite constatar que os valores estão abaixo do valor mínimo aceitável para o desempenho normal dos sistemas de aspersão.

Tabela 2. Valores dos coeficientes de uniformidade* determinados acima e abaixo da superfície do solo para espaçamento entre aspersores de $18 \times 18 \mathrm{~m}$

\begin{tabular}{|c|c|c|c|c|c|c|}
\hline \multirow[t]{6}{*}{ Irrigação } & \multicolumn{6}{|c|}{ Coeficientes de uniformidade $(\%)$} \\
\hline & \multirow{2}{*}{\multicolumn{2}{|c|}{$\begin{array}{l}\text { Antes da } \\
\text { Irrigação }\end{array}$}} & \multirow{2}{*}{\multicolumn{4}{|c|}{$\begin{array}{l}\text { Depois da } \\
\text { Irrigação }\end{array}$}} \\
\hline & & & & & & \\
\hline & \multicolumn{2}{|c|}{ Abaixo da } & \multicolumn{2}{|c|}{ Acima da } & \multicolumn{2}{|c|}{ Abaixo da } \\
\hline & \multicolumn{2}{|c|}{ Superfície } & \multicolumn{2}{|c|}{ Superfície } & \multicolumn{2}{|c|}{ Superfície } \\
\hline & CUC & CUD & CUC & CUD & CUC & CUD \\
\hline 1 & 94,62 & 90,72 & 72,90 & 57,16 & 91,62 & 86,20 \\
\hline 2 & 95,30 & 93,48 & 75,11 & 58,23 & 93,60 & 89,10 \\
\hline 3 & 94,88 & 93,50 & 75,00 & 57,12 & 93,57 & 86,48 \\
\hline 4 & 96,43 & 94,10 & 75,44 & 59,43 & 90,82 & 89,91 \\
\hline 5 & 96,05 & 94,41 & 74,72 & 60,12 & 91,93 & 88,06 \\
\hline 6 & 94,10 & 89,63 & 75,81 & 61,40 & 93,46 & 87,23 \\
\hline $\bar{X}$ & $95,23 b$ & $92,64 a$ & $74,83 \mathrm{c}$ & $58,91 d$ & $92,50 \mathrm{a}$ & $87,83 \mathrm{e}$ \\
\hline $\mathrm{CV}$ & 0,93 & 2,13 & 1,36 & 2,91 & 1,30 & 1,64 \\
\hline
\end{tabular}

*Valores médios seguidos da mesma letra não diferem estatisticamente a nível de 5\%.

Abaixo da superfície do solo o comportamento é semelhante ao observado para o menor espaçamento destacando-se, porém, os valores mais baixos dos coeficientes. Para este maior espaçamento, o teste " $t$ " revela que apenas as médias de CUD antes e CUC depois da irrigação, abaixo da superfície, não diferem estatisticamente a nível de 5\%; novamente o CUC, pouco sensível aos valores extremos, varia menos entre antes e após cada irrigação que o CUD. Ao contrário do ocorrido para o menor espaçamento, a diferença entre médias de CUC é significativa a 5\%, nestas condições, e o maior espaçamento dá origem a regiões que recebem pouca água, como atestam os coeficientes de acima da superfície. Estas regiões, doze horas após, embora tenham recebido alguma umidade via redistribuição, ainda estão mais secas; estes valores são os selecionados para o cálculo do CUD, o qual registra, portanto, redução acentuada entre antes e após a irrigação. Como a irregularidade da aplicação é grande, a suavização abaixo da superfície é mais evidente que no menor espaçamento, como mostram os valores de CUD na Tabela 2, onde a média de acima da superfície é de apenas $58,91 \%$ e, abaixo da superfície, após a irrigação, já atinge 87,83\%. Com o passar do tempo, a redistribuição promove maior uniformidade da umidade, o que está expresso pela média de valores de CUD antes das irrigações, de $93 \%$.

$\mathrm{Na}$ Tabela 3 são apresentados valores médios dos coeficientes de uniformidade obtidos para o espaçamento entre aspersores,

Tabela 3. Comparação entre valores médios* dos coeficientes de uniformidade (\%) obtidos para os dois espaçamentos entre aspersores, após as irrigações

\begin{tabular}{ccc}
\hline \multirow{2}{*}{ Coeficientes } & \multicolumn{2}{c}{ Espaçamentos entre Aspersores } \\
\cline { 2 - 3 } & $12 \times 12 \mathrm{~m}$ & $18 \times 18 \mathrm{~m}$ \\
\hline CUC - Acima & $88,23 \mathrm{a}$ & $74,83 \mathrm{~b}$ \\
CUC - Abaixo & $95,44 \mathrm{c}$ & $92,50 \mathrm{~d}$ \\
CUD - Acima & $82,14 \mathrm{~A}$ & $58,91 \mathrm{~B}$ \\
CUD - Abaixo & $91,83 \mathrm{C}$ & $87,83 \mathrm{D}$ \\
\hline
\end{tabular}

*Valores seguidos da mesma letra não diferem entre si ao nível de 1\% 
de $12 \times 12 \mathrm{~m}$ e $18 \times 18 \mathrm{~m}$, acima e abaixo da superfície do solo, após as irrigações. O maior espaçamento corresponde aos menores valores dos coeficientes, indicando menor uniformidade acima e abaixo da superfície.

Principalmente acima, mas também abaixo da superfície do solo, a redução do CUD em função do espaçamento é mais acentuada que a redução do CUC, evidenciando a maior sensibilidade deste coeficiente aos valores extremos inferiores. $\mathrm{O}$ teste " $\mathrm{t}$ " revela que os valores médios de CUC diferem estatisticamente a nível de $1 \%$ entre espaçamentos e entre acima e abaixo da superfície do solo; o mesmo ocorre para os valores de CUD.

O espaçamento de $12 \mathrm{~m}$ atende aos critérios usuais de uniformidade acima da superfície do solo, podendo ser adotado para este aspersor. $\mathrm{O}$ espaçamento de $18 \mathrm{~m}$ não poderia ser adotado segundo esses critérios, porém, mesmo o CUD revela que a uniformidade de umidade no perfil do solo é relativamente alta, poucas horas após o final da irrigação; tal fato sinaliza que a procura por sistemas de menor custo deve levar em conta a uniformidade abaixo da superfície do solo e não apenas acima da mesma, como é feito tradicionalmente nos projetos de sistemas de irrigação.

\section{CONCLUSÕES}

Com base nos resultados obtidos, pode-se concluir que:

1. Para ambos os coeficientes empregados, os valores dos coeficientes de uniformidade acima da superfície do solo foram menores que abaixo dela, mostrando que a redistribuição promove maior uniformidade da umidade no perfil do solo que a identificada para a lâmina aplicada.

2. O CUD é mais sensível aos valores extremos que o CUC e apresentou valores menores, nas condições estudadas, sugerindo que o CUD é mais adequado para a avaliação da uniformidade acima e abaixo da superfície.

3. O maior espaçamento acarreta menor uniformidade acima e abaixo da superfície do solo, mas a uniformidade abaixo da superfície mostra que esta irrigação atenderia aos critérios usualmente adotados, ao contrário do identificado acima da superfície. Quando o propósito é o dimensionamento econômico de sistemas, a uniformidade abaixo da superfície deve ser considerada.

\section{REFERÊNCIAS BIBLIOGRÁFICAS}

ABNT-ASSOCIAÇÃO BRASILEIRA DE NORMAS TÉCNICAS. Sistema de irrigação por aspersão pivôcentral, caracterização de desempenho do método de ensaio; projeto de normas. Rio de Janeiro, 22p, 1985. (12:02.08.005)
CHRISTIANSEN, E.J. Irrigation by sprinkling. Berkeley: University of California, 1942. 142p.(Bulletttin,670).

HART,W.E.; REYNOLDS, W.N. Analytical design of sprinkler systems. Transactions of the ASAE, St. Joseph, v.8, n.10, p-83-89, 1965.

HEERMANN, D.F.; DUKE, H.R.; SERAFIM, A.M.; DAWSON, L.L. Distribution functions to represent centerpivot water distribution. Transactions of the ASAE, St. Joseph, v.35, n.5, p.1465-1472, 1992.

JENSEN, M.E.; SWARNER, L.R.; PHELAN, J.T. Improving irrigation efficients. In. HAGAN, R.M. Irrigation of agriculture lands. St. Joseph: ASAE, 1967. Cap.61.p.112042(Agronomy,11).

KELLER. J. Sprinkler irrigation. Logan: Utah State University, 1984. 621p.

LOPEZ, E.M. Riego por aspersion. Petrolina: Centro de treinamento em irrigação. SUDENE/MINTER-IICA, 1975.33p

MANTOVANI, E.C.; VILLALOBOS, F.J.; ORGAZ,F.; FERERES, E. Modelling the effects of sprinkler irrigation uniformity on crop yield. Agricultural Water Management, Amsterndam, v.27,n.3/4, p.243-257, 1995.

OLITTA, F.L. Os métodos de irrigação. São Paulo: Nobel, 1981. 267p.

PAIVA, J.B.D. de. Uniformidade de aplicação de água, abaixo da superfície do solo, utilizando-se irrigação por aspersão. São Carlos: USP, 1980. 125p. Dissertação Mestrado

PERI,G.; HART, W.E.; NORUM, D.J Optimal irrigation depths-a method of analysis. Journal of the Irrigation and Drainage Division. ASCE, New York, v.105 n.4 34355,dec. 1979.

PERRENS, S.J. Numerical analysis of soil water uniformity under sprinkler irrigation. Journal Agricultural Engineering Research, Cambridge, v-30: p23-7, 1984.

REZENDE, R.; FRIZZONE; J.A.; BOTREL, T.B. Desempenho de um sistema de irrigação pivô-central quanto à uniformidade de distribuição de água, abaixo e acima da superfície do solo. Engenharia na Agricultura, Viçosa, v.2, n.19, p.01-07, 1992.

SCALOPPI, E.J. Exigência de energia para irrigação. Irrigação e Tecnologia Moderna, Brasília, v.21, p.13-17, 1985.

TARJUELO, J.M.; De JUAN, J.A.; VALIENTE, M.; GARCIA, P. Model for optimal cropping patterns within the farm based on crop water production functions and irrigation uniformity. II: A case study of irrigation scheduling in Albcete, Spain. Agricultural Water Management, Amsterdam, v.31, n.1/ 2, p.145-163, 1996.

WALKER, W.R. Explicit sprinkler irrigation uniformity: efficiency model. Journal of the Irrigation and Drainage Division. ASCE, New York, v.105, n.2, p.129-36, june 1979. 